\title{
Amplitude ratios and the approach to bulk criticality in parallel plate geometries
}

\author{
M. M. Leite \\ Instituto de Física Teórica, Universidade Estadual Paulista, 01405-900 São Paulo, SP, Brazil \\ M. Sardelich and M. D. Coutinho-Filho \\ Laboratório de Física Teórica e Computacional, Departamento de Física, Universidade Federal de Pernambuco,
} 50670-901 Recife, PE, Brazil

(Received 2 September 1998)

\begin{abstract}
We present analytical and numerical results for the specific heat and susceptibility amplitude ratios in parallel plate geometries. The results are derived using field-theoretic techniques suitable to describe the system in the bulk limit, i.e., $\left(L / \xi_{ \pm}\right) \gg 1$, where $L$ is the distance between the plates and $\xi_{ \pm}$is the correlation length above $(+)$ and below $(-)$ the bulk critical temperature. Advantages and drawbacks of our method are discussed in the light of other approaches previously reported in the literature. [S1063-651X(99)04703-0]

PACS number(s): 64.10.+h, 64.60.Fr, 64.60.Ak
\end{abstract}

\section{INTRODUCTION}

Since the advent of modern scaling concepts and renormalization-group techniques the study of finite-size and surface effects on the behavior of systems near or at criticality has attracted the attention of a number of investigators [1].

Fixing our interest in the case of a system confined between two infinite $(d-1)$-dimensional parallel plates distant $L$ from each other, we may classify three well defined distinct regimes in this problem. The first one, where the scaling variable $\left(L / \xi_{ \pm}\right) \gg 1$, is characterized by the dominance of bulk over surface and finite-size effects and the physics is quasi- $d$-dimensional. Here, $\xi_{ \pm}$specifies the critical correlation length above $(+)$ and below $(-)$ the bulk critical temperature $T_{c}$. The second regime, where $\left(L / \xi_{ \pm}\right) \ll 1$, the system behaves as a quasi- $(d-1)$-dimensional object. Finally, for $\left(L / \xi_{ \pm}\right) \sim 1$, the physics interpolates between a quasi- $d$ and a quasi- $(d-1)$-dimensional system. A full description of the system should therefore unveil very interesting crossover behaviors.

According to the region and phenomenon of interest, different field-theoretic techniques have been devised to deal with such systems. Indeed, Diehl and Dietrich $[2,3]$ successfully implemented these techniques to study critical and multicritical phenomena near surfaces within a finite momentum cutoff regularization scheme. The use of dimensional regularization was shown $[4,5]$ to simplify the computational procedure and allowed the study of ordinary [2] and special transitions [3] through standard $\phi^{4}$-field theories under Dirichlet and Neumann boundary conditions (DBC's and NBBC's), respectively. The former mimics very strong repulsive forces at the surface, thus preventing order at it (a parameter $c$, which measures these forces [2], has fixed point value $c^{*}=\infty$ ), whereas under the later boundary condition both the surface and the bulk go critical simultaneously. The special transition is in fact a multicritical point [3], $c^{*}=0$, where the two lines describing systems with repulsive $(c$ $>0)$ and attractive forces $(c<0)$ at the surface meet. In the later case, namely, the extraordinary transition $[5,6]$, the surface undergoes a second-order transition before criticality sets in the bulk. Moreover, it has been shown [7] that a scaling description holds so that the critical exponents associated with excess surface singularities may be expressed completely in terms of bulk exponents. However, it has also been shown [7] that fluctuations may induce divergences at the surface and in these cases local quantities and associated exponents must be defined resulting in new scaling relations. Therefore, in treating these quantities at the multicritical point, Neumann boundary conditions are valid only at the mean-field level [7].

On the other hand, in order to properly describe finite-size effects using field-theoretic techniques in critical systems subject, for example, to periodic boundary conditions (PBC's), Brezin and Zinn-Justin [8] and, independently, Rudnick, Guo, and Jasnow [9], introduced a method in which the zero-momentum component is isolated whereas the other nonzero modes are treated perturbatively. This method has been largely used [10-12] and generalized to study different boundary conditions. More recently [13], some difficulties regarding the treatment of critical systems below $T_{c}$ using this technique have been circumvented.

Both finite-size and surface effects are simultaneously present, except in special circumstances such as for PBC's, the surface effects of which vanish. At $T=T_{c}$, where Casimir forces are manifest, these contributions compete in a very special way, and powerful tools and methods such as conformal invariance [14] and elaborated perturbation techniques [15] have been used to study this regime and the approach to $T_{c}[12-15]$.

In this work we shall calculate specific heat and susceptibility amplitude ratios using field-theoretic and $\in$-expansion methods [16] particularly suitable to describe systems in the first regime mentioned above in which bulk behavior dominates over surface and finite-size contributions. The reported results complement previous studies [16] and shed some light on the approach to bulk criticality as the distance $L$ between the plates increases.

For a system of volume $V=A L$, where $A$ is a $(d-1)$-dimensional surface (layered geometry), the following asymptotic scaling form for the singular part of the freeenergy density holds [17]: 


$$
\begin{aligned}
f(|t|, L) \sim & \frac{1}{A L} Y\left(L|t|^{\nu / a}\right)=y_{b}|t|^{d \nu}+y_{s} \frac{|t|^{(d-1) \nu}}{L} \\
& +\delta f\left(L|t|^{\nu} / a\right)
\end{aligned}
$$

where $t=\left(T-T_{c}\right) / T_{c}, \quad \nu$ is the bulk correlation length exponent and $a$ is the only nonuniversal metric factor. Using the hyperscaling relation $d \nu=2-\alpha$, one identifies the first term (proportional to $y_{b}$ ) as the bulk contribution, the second one (proportional to $y_{s}$ ) as the excess surface term and the last one as a finite-size correction term. In the limit $\left(\xi_{ \pm} / L\right)$ $\ll 1$ one expects exponentially small corrections from $\delta f$, whereas for $\left(\xi_{ \pm} / L\right) \gg 1$ it compensates the bulk and surface contributions and gives rise to the Casimir effect [13-15] at $T=T_{c}$.

From the above scaling assumptions the specific heat and susceptibility should behave as

$$
\begin{aligned}
& C(t, L) \sim|t|^{-\alpha} A_{ \pm}\left(L|t|^{\nu} / a\right), \\
& \chi(t, L) \sim|t|^{-\gamma} C_{ \pm}\left(L|t|^{\nu / a}\right),
\end{aligned}
$$

where $\alpha$ and $\gamma$ are the bulk critical exponents, but even in the regime $L|t|^{\nu_{\gg} \gg 1}$ one expects that excess surface and finite-size contributions modify their critical amplitudes in a non-trivial manner. In fact, the ratio of these amplitudes are quite sensitive in identifying the universality class of a critical system, particularly in numerical simulations [18] where one has to control both corrections to scaling and surface and finite-size effects.

In Sec. II we explain our method and derive both the renormalized free energy and the equation of state from which the above quantities can be calculated. Finally, in Sec. III a discussion of the results and conclusions are presented.

\section{SPECIFIC HEAT AND SUSCEPTIBILITY CRITICAL AMPLITUDES}

In this section we shall use field-theoretic and renormalization-group techniques to calculate the amplitude ratios of $C$ and $\chi$ in layered geometries. We shall keep close contact with standard bulk $\phi^{4}$-field theory [19] and whenever necessary to deal with the finite size of the system we employ methods [16] which are particularly suitable in the regime $\left(L / \xi_{ \pm}\right) \gg 1$.

\section{A. Renormalized free energy and boundary conditions}

We start by writing the expression for the one-loop renormalized Helmholtz free-energy density at the fixed point associated with the bulk critical behavior of the system:

$$
\begin{aligned}
F(t, M ; L)= & \frac{1}{2} t M^{2}+\frac{1}{4 !} u^{*} M^{4}+\frac{1}{4}\left(t^{2}+u^{*} t M^{2}+\frac{1}{4} u^{*} M^{4}\right) I_{s p} \\
& +\frac{1}{2 L} \sum_{j} \int d^{d-1} q \ln [1+(1 / 2) \\
& \left.\times u^{*} M^{2} /\left(q^{2}+\kappa_{j}^{2}+t\right)\right] .
\end{aligned}
$$

In the equation above $t, M\left(t_{0}=\mathcal{Z}_{\phi^{2}} t, M_{0}=\mathcal{Z}_{\phi}^{1 / 2} M\right)$ are the renormalized (bare) reduced temperature and order param- eter, respectively, $\mathcal{Z}_{\phi^{2}}, \mathcal{Z}_{\phi}$ are renormalization functions, $u^{*}$ is the dimensionless renormalized coupling constant of a continuous (bulk) $\phi^{4}$ theory at the fixed point, $\vec{q}$ is a ( $d$ -1)-dimensional wave vector along the direction parallel to the plate, $\kappa_{j}=\pi j / L$ are the eigenvalues of the kinetic energy operator satisfying proper boundary conditions (see below) and $I_{s p}=\in^{-1}[1+(\in / 2)]+\mathcal{O}(\in)$ is the one-loop integral of a bulk $\phi^{4}$ theory evaluated at the symmetry point using dimensional regularization, where $\in=4-d$. Notice that taking the $\lim L \rightarrow \infty$ in Eq. (4) one obtains the standard expression for the $d$-dimensional $\phi^{4}$ one-loop renormalized free energy.

In deriving $F(t, M ; L)$ we have considered that the local field of a $\phi^{4}$ theory may satisfy periodic, Neumann, or Dirichlet boundary conditions, defined by $\phi(\vec{\rho}, z)=\phi(\vec{\rho}, z$ $+L),\left(\partial /\left.\partial z\right|_{z=0}\right) \phi(\vec{\rho}, z)=\left(\partial /\left.\partial z\right|_{z=L}\right) \phi(\vec{\rho}, z)=0, \quad$ and $\phi(\vec{\rho}, z=0)=\phi(\vec{\rho}, z=L)=0$, respectively, where $\vec{\rho}$ is a $(d$ $-1)$-dimensional position vector perpendicular to the $z(d$ th $)$ direction. It then follows that the sum in Eq. (4) has values $j=0, \pm 1, \pm 2, \ldots$, for PBC's, $j=0,1,2, \ldots$, for NBC's, and $j=1,2, \ldots$, for DBC's, respectively. The local field is Fourier transformed in the form [16]

$$
\phi(\vec{\rho}, z)=\sum_{j}(2 \pi)^{1-d} \int d^{d-1} q \exp (i \vec{q} \cdot \vec{\rho}) \phi_{j}(\vec{q}) u_{j}(z),
$$

where $\phi_{j}(\vec{q})$ are plane waves parallel to the plate and $u_{j}(z)$ are eigenfunctions of the kinetic energy operator $\left(-d^{2} / d z^{2}\right)$ with eigenvalues $\kappa_{j}^{2}$. The bare order parameter $M_{0}$ is thus the expectation value of the local field above. We call attention that the usual counterterms of a bulk $\phi^{4}$ theory are used to renormalize the free energy and that the boundary conditions are implemented on the bare vertex functions. Details of the Feynman rules involving propagators and vertices can be found in Ref. [16].

\section{B. Specific heat amplitude ratio}

Since the vertex function $\Gamma^{(0,2)}$ is additively renormalized, the critical behavior (singular part) of the specific heat is calculated using the expression [19]

$$
C=A|t|^{-\alpha}=-\frac{\nu}{\alpha} B\left(u^{*}\right)-\Gamma_{R}^{(0,2)},
$$

where $B\left(u^{*}\right)$ is the inhomogeneous term of the renormalization-group equation for $\Gamma_{R}^{(0,2)}$ and

$$
\Gamma_{R}^{(0,2)}=\frac{\partial^{2}}{\partial t^{2}} F(t, M ; L) .
$$

For $T>T_{c}, \quad M=0$, and we find, using Eqs. (4) and (7),

$$
\Gamma_{R}^{(0,2)}\left(T>T_{c}\right)=-\frac{1}{2 L} \sum_{j} \int \frac{d^{d-1} q}{\left(q^{2}+\kappa_{j}^{2}+t\right)^{2}}+\frac{1}{2} I_{s p},
$$

whereas for $T<T_{c}$ we use the value of $M$ at the coexistence curve, namely, $u^{*} M^{2}=-6 t$, to obtain 


$$
\Gamma_{R}^{(0,2)}\left(T<T_{c}\right)=-\frac{3}{u^{*}}-\frac{2}{L} \sum_{j} \int \frac{d^{d-1} q}{\left(q^{2}+\kappa_{j}^{2}+2|t|\right)^{2}}+2 I_{s p}
$$

The one-loop integrals are evaluated using dimensional regularization and some useful formulas $[16,20]$ to sum infinite series. We thus obtain for the boundary conditions of interest:

$$
\begin{aligned}
\frac{1}{L} \sum_{j} \int & \frac{d^{d-1} q}{\left(q^{2}+\kappa_{j}^{2}+\tilde{t}\right)^{2}} \\
= & \tilde{t}^{-\in / 2} \frac{1}{\in}\left(1-\frac{\epsilon}{2}\right)+2 \pi^{-1 / 2}\left(\frac{2 \pi}{L}\right)^{d-4} \Gamma(d / 2) \\
& \times \Gamma[(5-d) / 2)] \sin [\pi(5-d) / 2] f_{(5-d) / 2}\left[\frac{L \tilde{t}^{(5-d) / 2}}{2^{\sigma} \pi}\right] \\
& +\tau \frac{\pi^{1 / 2}}{2} \Gamma(d / 2) \Gamma[(5-d) / 2]\left[\tilde{t}^{(d-5) / 2} / L\right]
\end{aligned}
$$

where $S_{d} /(2 \pi)^{d} \equiv 1, \quad S_{d}$ being the area of the d-dimensional unity sphere, $\tilde{t}=t+(1 / 2) u^{*} M^{2}=t(\tilde{t}=2|t|)$ for $T>T_{c}\left(T<T_{c}\right), \quad \sigma=1$ for PBC's, $\sigma=0$ for both NBC's and DBC's, $\tau=0,+1,-1$ for PBC's, NBC's, and DBC's, respectively, and

$$
f_{\alpha}(a)=\int_{a}^{\infty} \frac{\left(u^{2}-a^{2}\right)^{-\alpha} d u}{\exp (2 \pi u)-1}, \quad \alpha<1 .
$$

The above representation is particularly suitable in the bulk limit, $L / \xi_{ \pm} \gg 1$, but difficulties arise as $a \rightarrow 0 \quad\left(L / \xi_{ \pm} \ll 1\right)$ in Eq. (11), as will be later numerically evidenced. A representation allowing access to both regimes, but not without some problems for $T<T_{c}$, was formulated by Krech and Dietrich [15] and used to study films at bulk criticality.

Now using the $\in$ expansion [19] for the nonsingular part of the specific heat, $-(\nu / \alpha) B\left(u^{*}\right)=\left(\frac{3}{2} \in\right)+(295 / 108)$ $+\mathcal{O}(\in)$, we find the amplitudes above and below $T_{c}$ :

$$
\begin{aligned}
A_{+}= & \frac{3}{\in 2}\left[1+\in \frac{47}{54}+\in \frac{2^{2-\sigma}}{3} f_{1 / 2}\left(\frac{L t^{1 / 2}}{2^{\sigma} \pi}\right)+\in \frac{\tau}{3} \frac{\pi}{L t^{1 / 2}}\right] \\
& +\mathcal{O}\left(\epsilon^{2}\right), \\
A_{-}= & \frac{6}{\in 2^{\alpha}}\left[1-\in \frac{7}{54}+\in \frac{2^{2-\sigma}}{3} f_{1 / 2}\left(\frac{\sqrt{2} L|t|^{1 / 2}}{2^{\sigma} \pi}\right)\right. \\
& \left.+\in \frac{\tau \pi}{3 \sqrt{2} L|t|^{1 / 2}}\right]+\mathcal{O}\left(\in^{2}\right),
\end{aligned}
$$

where $\alpha=\in / 6+\mathcal{O}\left(\in^{2}\right)$.

From the above equations we finally obtain the specific heat amplitude ratio:

$$
\frac{A_{+}}{A_{-}}=\frac{2^{\alpha}}{4}\left[1+\in f(x)+\in S_{A}(x)\right]+\mathcal{O}\left(\in^{2}\right),
$$

where $f(x)$ and $S_{A}(x)$ are given by

$$
f(x)=\frac{2^{2-\sigma}}{3}\left[f_{1 / 2}\left(x / 2^{\sigma} \pi\right)-f_{1 / 2}\left(\sqrt{2} x / 2^{\sigma} \pi\right)\right]
$$

$$
S_{A}(x)=\tau \frac{\pi}{3 x}\left(1-\frac{1}{\sqrt{2}}\right)
$$

and $x=L / \xi$, with $\xi=|t|^{-1 / 2}$. For $x \rightarrow \infty$, we can use the asymptotic limit $[16,20]$ for $f_{1 / 2}(a)$ in Eq. (11) and write $f(x)$ in the more simplified form

$$
\begin{aligned}
f(x)= & \frac{2^{1-\sigma}(2 \pi)^{1 / 2}}{3}\left[\frac{\exp \left(-2^{1-\sigma} x\right)}{\left(2^{1-\sigma} x\right)^{1 / 2}}\right. \\
& \left.-\frac{\exp \left(-\sqrt{2} 2^{1-\sigma} x\right)}{\left(\sqrt{2} 2^{1-\sigma} x\right)^{1 / 2}}\right], \quad x \rightarrow \infty .
\end{aligned}
$$

\section{Susceptibility amplitude ratio}

Using Eq. (1) we obtain the following renormalized equation of state:

$$
\begin{aligned}
H_{R}= & \frac{\partial F}{\partial M}=t M+\frac{1}{6} u^{*} M^{3}+\frac{1}{2} u^{*} M\left(t+\frac{1}{2} u^{*} M^{2}\right) \\
& \times\left[I_{s p}-\sum_{j} \frac{1}{L} \int \frac{d^{d-1} q}{\left(q^{2}+\kappa_{j}^{2}\right)\left(q^{2}+\kappa_{j}^{2}+t+\frac{1}{2} u^{*} M^{2}\right)}\right] .
\end{aligned}
$$

The one-loop integral is calculated similarly as for the specific heat:

$$
\begin{aligned}
\frac{1}{L} \sum_{j} & \int \frac{d^{d-1} q}{\left(q^{2}+\kappa_{j}^{2}\right)\left(q^{2}+\kappa_{j}^{2}+\tilde{t}\right)} \\
= & \frac{1}{2} \Gamma[2-(\in / 2)] \Gamma(\in / 2) \int_{0}^{1} d x(\tilde{t} x)^{-\in / 2} \\
& +2 \pi^{-1 / 2}\left(\frac{2^{\sigma} \pi}{L}\right)^{d-4} \Gamma(d / 2) \\
& \times \Gamma[(5-d) / 2] \sin [\pi(5-d) / 2] \int_{0}^{1} d x f_{(5-d) / 2} \\
& \times\left[\frac{L(\tilde{t} x)^{(5-d) / 2}}{2^{\sigma} \pi}\right]+\tau \frac{\pi^{1 / 2}}{2} \Gamma(d / 2) \Gamma[(5-d) / 2] \\
& \times \int_{0}^{1} d x\left[(\tilde{t} x)^{(d-5) / 2} / L\right] .
\end{aligned}
$$

By noticing that the first term in the right-hand side of Eq. (19) may be written as $\left[\in^{-1}-(1 / 2) \ln \tilde{t}\right]$, and using the $\in$-expansion representation for $I_{s p}$, we obtain, to first order in $\in$, 


$$
\begin{aligned}
H_{R}= & t M+\frac{1}{6} u^{*} M^{3}+\frac{1}{4} u^{*} M \tilde{t}\{(1+\ln \tilde{t}) \\
& \left.-2 \int_{0}^{1} d y f_{1 / 2}\left[\frac{L(y \tilde{t})^{1 / 2}}{2^{\sigma} \pi}\right]+\tau \frac{\pi \tilde{t}^{-1 / 2}}{L}\right\},
\end{aligned}
$$

where $u^{*}=(2 / 3) \in+\mathcal{O}\left(\in^{2}\right)$ and $\tilde{t}=t+(1 / 2) u^{*} M^{2}$. from

The susceptibility amplitudes are then readily calculated

$$
\chi^{-1}=\left(C|t|^{-\gamma}\right)^{-1}=\Gamma_{R}^{(2,0)}=\frac{\partial H_{R}}{\partial M} .
$$

As before, for $T>T_{c}, \quad M=0$, and for $T<T_{c}$ we use $u^{*} M^{2}=-6 t$. The amplitudes above and below $T_{c}$ thus read

$$
\begin{gathered}
C_{+}=1-\frac{\epsilon}{6}+\frac{\epsilon}{3} \int_{0}^{1} d y f_{1 / 2}\left(\frac{x y^{1 / 2}}{2^{\sigma} \pi}\right)+\in \frac{\tau \pi}{6 x}+\mathcal{O}\left(\in^{2}\right), \\
C_{-}=\frac{1}{2}\left\{1-\frac{\epsilon}{6}(4+\ln 2)+\frac{\epsilon}{3} \int_{0}^{1} d y f_{1 / 2}\left[\frac{x(2 y)^{1 / 2}}{2^{\sigma} \pi}\right]\right. \\
\left.+\in \frac{\tau \pi}{6 \sqrt{2} x}\right\}+\mathcal{O}\left(\in^{2}\right),
\end{gathered}
$$

where $x=L / \xi$.

Using Eq. (11) and performing the integrations in $y$, we find the susceptibility amplitude ratio:

$$
\frac{C^{+}}{C_{-}}=2^{\gamma-1} \frac{\gamma}{\beta}+\in h(x)+\in S_{C}(x)+\mathcal{O}\left(\in^{2}\right),
$$

where $\quad \gamma=1+(\in / 6)+\mathcal{O}\left(\in^{2}\right), \quad \beta=1 / 2-(\in / 6)+\mathcal{O}\left(\in^{2}\right)$ and

$$
\begin{gathered}
h(x)=\frac{2^{\sigma+1} \pi\left[\frac{(1-\sqrt{2})}{3 x}-\int_{a}^{\infty} d u g(a / u)\right.}{\left.+\sqrt{2} \int_{b}^{\infty} d u g(b / a)\right],} \\
S_{C}(x)=\tau \frac{\pi}{3 x}\left(1-\frac{1}{\sqrt{2}}\right),
\end{gathered}
$$

with $a=\sqrt{2} x / 2^{\sigma} \pi, \quad b=x / 2^{\sigma} \pi$, and

$$
g(c / u)=\frac{u \cos [\arcsin (c / u)]}{\exp (2 \pi u)-1} .
$$

\section{DISCUSSION AND CONCLUSIONS}

First, we should point out that the main step in our approach is the representation $[16,20]$ used to evaluate the discrete sums in Eqs. (10) and (19). It has proved very useful in different field-theoretic contexts [20] and here it clearly helps to split the bulk, surface and finite size contributions, as required by scaling [see Eqs. (1)-(3)], in a rather simple way,

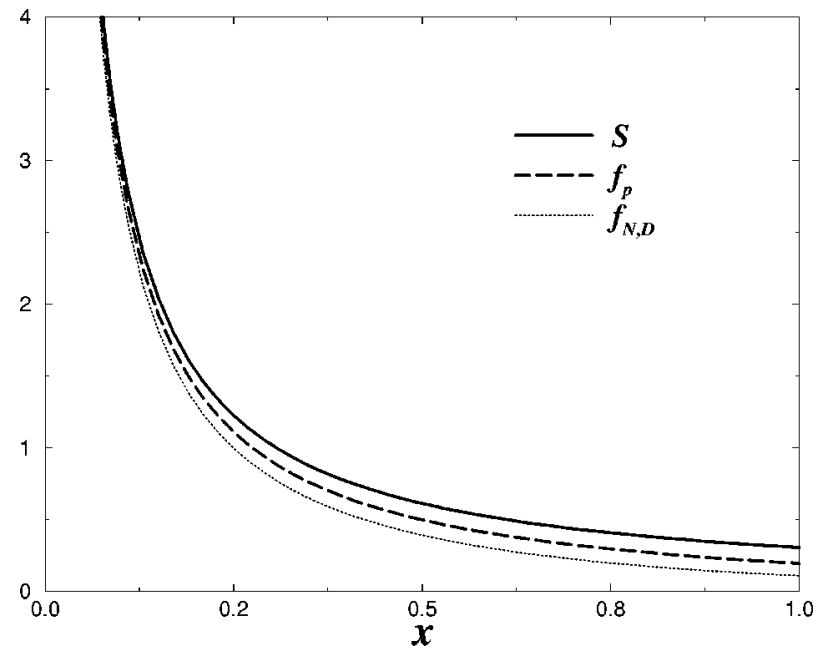

FIG. 1. Amplitude of the excess surface contribution (solid line), from Eqs. (16) and (26), and scaling functions $f_{p}$ (dashed line) and $f_{N, D}$ (dotted line) for periodic, Neumann, and Dirichlet boundary conditions, respectively, numerically evaluated using Eqs. (15) and (11) in $d=3$, as functions of $x=L / \xi$.

though its range of effectivenes precludes direct access to the Casimir effect.

Second, our starting renormalized free energy, Eq. (4), does not consider any distortion of the order parameter profile, i.e., our description is restricted to calculating the effect of the boundary conditions on bulk quantities as a result of fluctuations, i.e., in the amplitude ratios, Eqs. (14) and (24), excess surface and finite size contributions are of $\mathcal{O}(\in)$. Nevertheless, we observe that if the excess surface contributions for NBC's in Eqs. (12) and (13) are isolated, we find $\left(A_{+} / A_{-}\right)_{s}=2^{-3 / 2}+\mathcal{O}(\in)$, which is the same result derived in Ref. [5] for the special transition (the excess surface specific heat exponent is $\alpha_{S}=\alpha+\nu$ ). This is so because in this particular case there is no distortion of the order parameter at the mean-field level. Notice also that, above $T_{c}, \quad\left(A_{+}\right)_{s p} /\left(A_{+}\right)_{o r d}=-1+\mathcal{O}\left(\in^{2}\right)$, where $\left(A_{+}\right)_{s p, \text { ord }}$ refer to the specific heat amplitudes at the special (NBC's) and ordinary (DBC's) transitions, a result already derived in Ref. [4]. As for the excess surface contributions for the suscepti

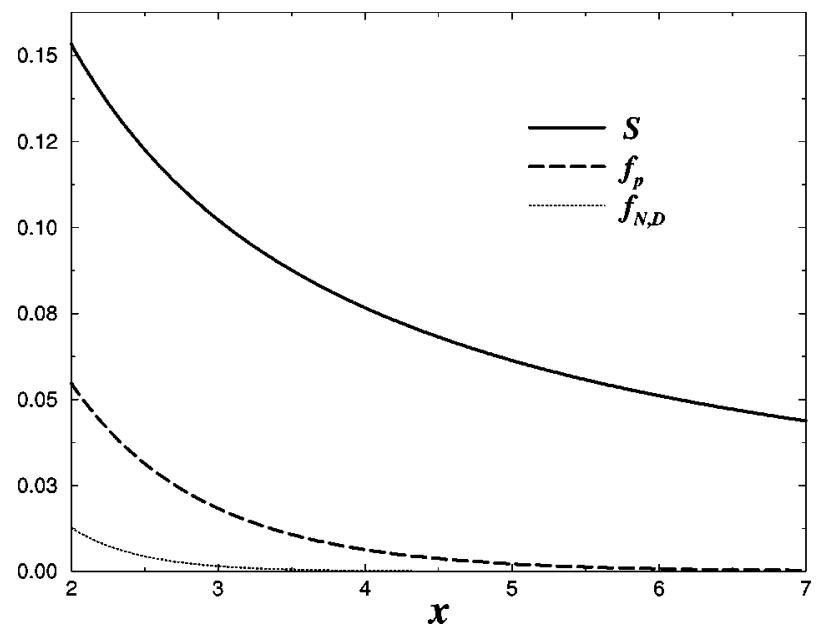

FIG. 2. Same as in Fig. 1 for $2 \leqslant x \leqslant 7$. 


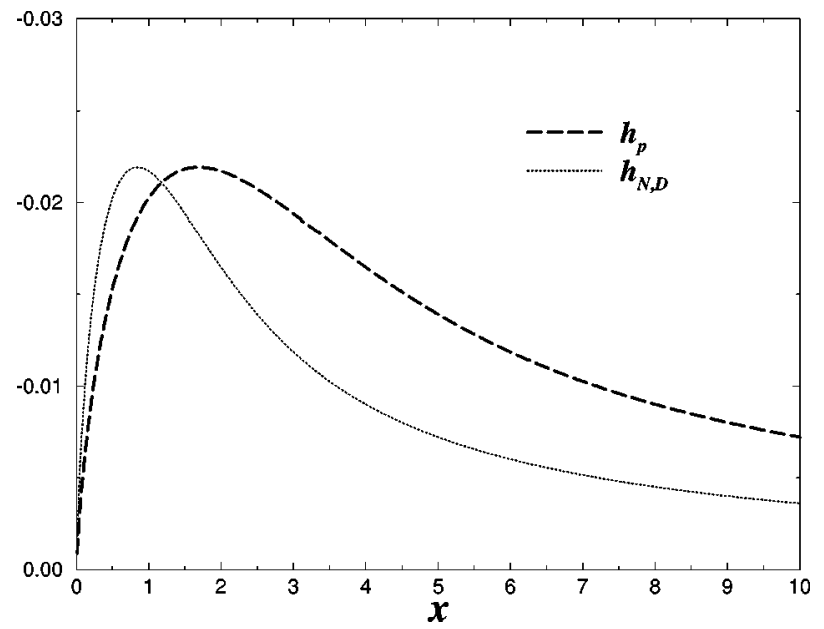

FIG. 3. Scaling functions $h_{p}$ (dashed line) and $h_{N, D}$ (dotted line) for periodic, Neumann, and Dirichlet boundary conditions, respectively, numerically evaluated using Eqs. (25) and (27) in $d=3$, as functions of $x=L / \xi$.

bility amplitudes we also notice that the last term in Eq. (22) is consistent with the result found in Ref. [7] for the special transition above $T_{c}\left(\gamma_{S}=\gamma+\nu\right)$, since again no distortion of the order parameter is necessary in this case. Below $T_{c}$, however, the order parameter profile differs from the bulk value as one approaches the surface of the plate, and therefore no comparasion can be made since our method excludes this feature as a starting point.

From the discussion above and the derived results in Sec. II, particularly Eqs. (14)-(17) and (24)-(27), it is clear that in the regime $L / \xi_{ \pm} \gg 1$, and to first order in an $\in$ expansion, the specific heat and susceptibility display singularities well described by bulk exponents, but with amplitudes sensitive to the boundary conditions, which manifest as excess surface and finite-size contributions. Notice also that these fluctuation effects result quite effectively from the difference between the amplitudes of the correlation length above and below $T_{c}$, which satisfy $\left(\xi_{0,+} / \xi_{0,-}\right)=\sqrt{2}+\mathcal{O}(\in)$.

In Figs. 1, 2, and 3 we plot the scaling functions $f(x)$ and $h(x)$, numerically evaluated in $d=3$, as defined by Eqs. (11),(15),(25)-(27). They both decay very rapidly to zero as $x=L t^{1 / 2}$ increases, in agreement with the asymptotic result $[16,20]$ for $f_{1 / 2}(x), \quad x \rightarrow \infty$. In fact, for PBC's and $x=7$ we find $f_{p}(x) \simeq 2.7 \times 10^{-4}$ either by using the numerical estimate or the asymptotic result predicted by Eq. (17). For this value of $x$ it is indeed expected [18] that these corrections to the bulk limit are indeed negligible. Notice also that for DBC's and NBC's the magnitude of the scaling functions are the same in our one-loop approximation, in agreement with Ref. [15], although a two-loop calculation shows [15] that they differ slightly if the same regime of validity applies. However, as $x \rightarrow 0$ our approach does not correctly describe the Casimir effect, as shown in Figs. 1 and 2: $f(x)$ diverges and $h(x)$ approaches zero, whereas in a correct treatment [13-15] both tend to a constant value, the Casimir amplitudes for each case. This failure for $x<1$ has already been pointed out by Nemirovsky and Freed [16]. Here, our results

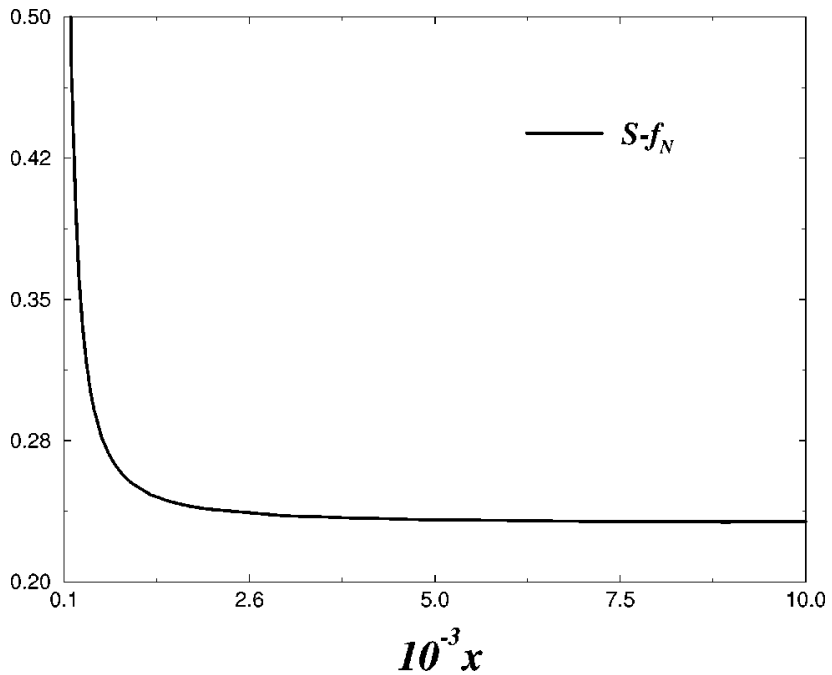

FIG. 4. Difference (apart from a minus sign) between the excess surface contribution and the scaling function $f_{N}$ for the Neumann boundary condition as a function of $x=L / \xi$.

deal with this issue in a more quantitative way, thus evidencing the advantages, limitations and drawbacks of the method.

For comparison, we also plot in Fig. 1 the excess surface contribution for the cases of both NBC's and DBC's, i.e., $\left|S_{A}(x)\right|=\left|S_{C}(x)\right| \equiv S(x)$. As clearly seen from Figs. 1 and 2, as $x \rightarrow \infty$ these contributions are the leading ones $[11,15]$ modifying the amplitudes of both the bulk specific heat and susceptibility. Notice that they have the same magnitude and decay as $x^{-1}$ [this is fortuitously true only because these effects are treated as fluctuation contributions to the bulk limit, see Eqs. (16),(26)].

Finally, in Fig. 4 we plot the difference between the excess surface contribution and the scaling function for NBC's. We see that this difference "almost" saturates as $x \rightarrow 0$, as expected in the Casimir effect, but lastly it diverges for very small values of $x$ (an $x^{-1}$ dependence is in fact expected from Eq. (11) as $x \rightarrow 0$, but an extra $\ln x$ contribution precludes a good description of the Casimir effect).

In summary, we have presented a field-theoretic description of the approach to bulk criticality in parallel plate geometries, in which excess surface and finite-size contributions appear as a result of fluctuations and are controlled by the boundary condition imposed on the system. Despite the fact that other more general methods to deal with finite systems do exist, our approach is probably the simplest one and a fair description, at least to $\mathcal{O}(\in)$, in the regime $L / \xi_{ \pm}$ $\gg 1$.

\section{ACKNOWLEDGMENTS}

We thank A. M. Nemirovsky for collaboration in an early stage of this work and several fruitful discussions. M. M. L. acknowledges FAPESP (State of São Paulo Foundation) for financial support under Grant No. 96/ 03546-7. M.D.C.-F. and M.S. acknowledge FINEP, CNPQ, CAPES, and FACEPE (Brazilian agencies) for financial support. 
[1] See, e.g., M.E. Fisher, in Critical Phenomena, edited by M. S. Green (Academic, London, 1971), p. 1; V. Privman, in Finite Size Scaling and Numerical Simulation of Statistical Systems, edited by V. Privman (World Scientific, Singapore, 1990), p. 1; M. N. Barber, in Phase Transitions and Critical Phenomena, edited by C. Domb and J. L. Lebowitz (Academic, New York, 1983), Vol. VIII, p. 145; H. W. Diehl, in Phase Transitions and Critical Phenomena, edited by C. Domb and J. L. Lebowitz (Academic, New York, 1986), Vol. X, p. 75; S. Dietrich, in Phase Transitions and Critical Phenomena, edited by C. Domb and J. L. Lebowitz (Academic, New York, 1988), Vol. XII, p. 1.

[2] H. W. Diehl and S. Dietrich, Z. Phys. B 42, 65 (1981); 43, 281(E) (1981); Phys. Lett. A 80, 408 (1980).

[3] H. W. Diehl and S. Dietrich, Z. Phys. B 50, 117 (1983).

[4] Y. Y. Goldschmidt and D. Jasnow, Phys. Rev. B 29, 3990 (1984).

[5] E. Eisenriegler, J. Chem. Phys. 81, 4666 (1984); E. Eisenriegler and P. J. Upton, ibid. 98, 3582(E) (1993).

[6] P. Upton, Phys. Rev. B 45, 8100 (1992); E. Eisenriegler and M. Stapper, ibid. 50, 10009 (1994).

[7] H. W. Diehl, G. Gompper, and W. Speth, Phys. Rev. B 31, 5841 (1985); H. W. Diehl and M. Shpot, Phys. Rev. Lett. 73, 3431 (1994). See also H. W. Diehl, in Phase Transitions and Critical Phenomena (Ref. [1]).

[8] E. Brézin and J. Zinn-Justin, Nucl. Phys. B 257, 867 (1985).

[9] J. Rudnick, H. Guo, and D. Jasnow, J. Stat. Phys. 41, 353 (1985)

[10] E. Eisenriegler and R. Tomaschitz, Phys. Rev. B 35, 4876 (1987); E. Eisenriegler, Z. Phys. B 61, 299 (1985).

[11] W. Huhn and V. Dohm, Phys. Rev. Lett. 61, 1368 (1988); V.
Dohm, Phys. Scr. T49, 46 (1993) and references therein.

[12] For crossover effects, see: D. O'Connor and C. R. Stephens, Nucl. Phys. B 360, 297 (1991); J. Phys. A 25, 101 (1992); Phys. Rev. Lett. 72, 506 (1994).

[13] X. S. Chen, V. Dohm, and N. Schultka, Phys. Rev. Lett. 77, 3641 (1996); A. Esser, V. Dohm, and X. S. Chen, Physica A 222, 355 (1995) and references therein.

[14] J. L. Cardy, Phys. Rev. Lett. 65, 1443 (1990); T. W. Burkhardt and T. Xue, ibid. 66, 895 (1991); E. Eisenriegler and U. Ritschel, Phys. Rev. B 51, 13717 (1995); T. W. Burkhardt and E. Eisenriegler, Phys. Rev. Lett. 74, 3189 (1995).

[15] M. Krech and S. Dietrich, Phys. Rev. Lett. 66, 345 (1991); 67, 1055(E) (1991); Phys. Rev. A 46, 1886 (1992); E. Eisenriegler, M. Krech, and S. Dietrich, Phys. Rev. Lett. 70, 619 (1993); 70, 2051(E) (1993); M. Krech, Phys. Rev. E 56, 1642 (1997); The Casimir Effect in Critical Systems (World Scientific, Singapore, 1994), and references therein.

[16] A. M. Nemirovsky and K. F. Freed, Nucl. Phys. B 270, 423 (1986); J. Phys. A 18, L319 (1985); M. M. Leite, A. M. Nemirovsky, and M. D. Coutinho-Filho, J. Magn. Magn. Mater. 104-107, 181 (1992).

[17] See, e.g. V. Privman and M. E. Fisher, Phys. Rev. B 30, 322 (1984); V. Privman, ibid. 38, 9261 (1988).

[18] M. Caselle and M. Hasenbusch, J. Phys. A 30, 4963 (1997); X. S. Chen, V. Dohm, and A. L. Talapov, Physica A 232, 375 (1996).

[19] See, e.g., D.J. Amit, Field Theory, the Renormalization Group and Critical Phenomena (World Scientific, Singapore, 1984).

[20] See, e.g., N. D. Birrell and L. H. Ford, Phys. Rev. D 22, 330 (1980); D. J. Toms, ibid. 21, 928 (1980). 